\title{
Clinicopathological analysis of diffuse large B-cell lymphoma lacking surface immunoglobulin light chain restriction on flow cytometry
}

\author{
Yosuke Nakaya, ${ }^{1)}$ Miho Sakaida, ${ }^{2)}$ Masahiro Yoshida, ${ }^{1)}$ Katsujun Shimizu, ${ }^{1)}$ Naoko Yagi, ${ }^{1)}$ \\ Minako Tsutsumi, ${ }^{1)}$ Takuro Yoshimura, ${ }^{1)}$ Yoshiki Hayashi, ${ }^{1)}$ Takafumi Nakao, ${ }^{1)}$ Takeshi Inoue, ${ }^{2)}$ \\ Takahisa Yamane ${ }^{1)}$
}

\begin{abstract}
Although diffuse large B-cell lymphoma (DLBCL) occasionally lacks surface immunoglobulin light chain restriction (iLCR) on flow cytometry (FCM), little evidence is available for iLCR-negative DLBCL. We retrospectively compared clinicopathological features of iLCR-positive and iLCR-negative DLBCL diagnosed at our institute between April 2007 and March 2018. iLCR-positive was defined as a $\kappa / \lambda$ ratio less than 0.5 or greater than 3 in the gated population on dual-color FCM, and iLCRnegative as other values. Of 81 DLBCL cases with available immunophenotyping by FCM, 63 iLCR-positive DLBCL (78\%) and 18 iLCR-negative DLBCL (22\%) cases were identified. Survival outcomes of patients with iLCR-negative DLBCL were comparable with those of patients with iLCR-positive DLBCL. Pathological analysis revealed no significant difference except for the lower expression of BCL6 in iLCR-negative DLBCL $(12.5 \%$ vs $65.5 \%, \mathrm{p}<0.001)$, although there was a slightly higher frequency of necrosis $(47.1 \%$ vs $20.7 \%, p=0.058)$ and lower expression of CD10 $(11.8 \%$ vs $35.0 \%, p=0.078)$ in iLCR-negative DLBCL than in iLCR-positive DLBCL. The underlying mechanism remains unclear; however, low expression of germinal center markers and tumor necrosis may be associated with the loss of iLCR in DLBCL.
\end{abstract}

Keywords: diffuse large B-cell lymphoma, flow cytometry, immunoglobulin light chain restriction, necrosis, cell of origin

\section{INTRODUCTION}

Flow cytometry (FCM) is a widespread and useful technique that rapidly analyzes the immunophenotype of hematological neoplasms. ${ }^{1}$ Although the detection of surface immunoglobulin light chain restriction (iLCR) by FCM reflects the monoclonality of mature B-cell lymphoma, hematologists and pathologists sometimes encounter cases of histologically confirmed malignant B-cell lymphoma lacking iLCR. Diffuse large B-cell lymphoma (DLBCL) is the most common subtype of B-cell non-Hodgkin lymphoma (NHL) accounting for $30 \%-50 \%$ of all adult NHL. ${ }^{2}$ Previous reports demonstrated that $7 \%-19 \%$ of B-cell NHL and $22 \%-$ $30 \%$ of DLBCL do not exhibit iLCR on FCM. ${ }^{3-6}$ The loss of iLCR can be explained by (i) the lack of or decreased expression of surface immunoglobulin light chain on lymphoma cells; (ii) frequent cell necrosis, apoptosis, and mitosis (pathological features associated with the high proliferation of tumor cells); or (iii) sampling or mechanical errors during the procedure of FCM. ${ }^{6-9}$ However, little evidence is available for the clinical prognosis associated with pathological features in iLCR-negative DLBCL.

Our study examined the clinicopathological features of iLCR-negative DLBCL in comparison with iLCR-positive DLBCL treated mainly with rituximab-containing multiagent chemotherapy.

\section{MATERIALS AND METHODS}

\section{Patient selection}

We retrospectively reviewed the medical records and selected consecutive patients who underwent dual-color FCM and were newly diagnosed with DLBCL at Osaka City General Hospital between April 2007 and March 2018. Clinical data, including sex, the International Prognostic

\footnotetext{
Received: May 3, 2021. Revised: August 10, 2021. Accepted: September 2, 2021. J-STAGE Advance Published: October 26, 2021 DOI: $10.3960 /$ jslrt. 21017

${ }^{1)}$ Department of Hematology, Osaka City General Hospital, Osaka, Japan, ${ }^{2}$ Department of Pathology, Osaka City General Hospital, Osaka, Japan

Corresponding author: Masahiro Yoshida, Department of Hematology, Osaka City General Hospital, 2-13-22 Miyakojima-hondori, Miyakojima-ku, Osaka City, Osaka, 534-0021,

Japan. E-mail: yoshidamasahiro1009@gmail.com

Copyright $(\subset 2021$ The Japanese Society for Lymphoreticular Tissue Research

(c) BY-NC-SA This work is licensed under a Creative Commons Attribution-NonCommercial-ShareAlike 4.0 International License.
} 
Index (IPI) factors (serum lactate dehydrogenase, performance status (PS), Ann Arbor staging classification, and extranodal lesions), ${ }^{10} \mathrm{~B}$-symptoms, and presence of a bulky mass at initial diagnosis were collected from the medical charts. Patients with human immunodeficiency virus infection, central nervous system involvement, intravascular large B-cell lymphoma, primary mediastinal large B-cell lymphoma, and transformation from indolent B-cell lymphoma were excluded. As a general rule of initial treatment, patients younger than 70 years received six cycles of R-CHOP (rituximab, cyclophosphamide, doxorubicin, vincristine, and prednisone) and those over 70 years received six cycles of dosereduced R-THP-COP (rituximab, pirarubicin, doxorubicin, vincristine, and prednisone) chemotherapy. ${ }^{11,12}$ Upfront autologous stem cell transplantation and radiotherapy were not incorporated regardless of the IPI or presence of a bulky mass.

\section{Flow cytometry}

Dual-color FCM was performed on fresh samples according to the protocol of $\mathrm{CD} 45 /$ side-scatter gating using antibodies against $\mathrm{CD} 5, \mathrm{CD} 10, \mathrm{CD} 19, \mathrm{CD} 20, \mathrm{CD} 23, \mathrm{CD} 25, \mathrm{CD} 30$, $\kappa$-chain, and $\lambda$-chain. ${ }^{13}$ iLCR-positive was defined as a $\kappa / \lambda$ ratio in the gated population of less than 0.5 or greater than 3 , and iLCR-negative as other values. ${ }^{14}$ Patients whose proportion of both CD19-positive and CD20-positive cells in the gated population was less than $50 \%$ were excluded because this proportion did not reflect DLBCL due to the low inclusion of tumor cells resulting from a sampling error or gating error caused by CD45 negativity on lymphoma cells.

\section{Histopathological and immunochemical analyses}

The biopsy tissue samples were fixed with $10 \%$ neutralbuffered formalin, embedded in paraffin, cut into $3-\mu \mathrm{m}$-thick sections, and stained with hematoxylin and eosin, and routine antibodies for immunostaining, including CD20, CD10, BCL6, MUM1, BCL2, and MYC. Pathological diagnosis was conducted by three pathologists based on the revised fourth edition of the World Health Organization (WHO) classification. ${ }^{15}$ Histological features, including the morphological variants, number of nucleoli, cellularity of lymphoma cells, presence of focal or geographic necrosis, and degree of apoptosis and mitosis were investigated in both iLCR-positive and iLCR-negative DLBCL. Cellularity was defined as high, $>75 \%$ of cells in sample were neoplastic; or moderate, $\leq 75 \%$ neoplastic cells. The degree of apoptosis and mitosis was as follows: absent, not observed in sample; occasional, scattered in several fields; or abundant, observed throughout most fields. Subclassification of cellular origin was determined by the immunohistochemical algorithm developed by Hans et al. ${ }^{16}$ Cases were scored positive for CD10, BCL6, and MUM1 if $30 \%$ or more neoplastic cells were stained with the antibodies. ${ }^{16}$ The cut-off levels for BCL2 and MYC were $50 \%$ and $40 \%$ in accordance with recent reports. ${ }^{15}$ Immunostaining for immunoglobulin light chains ( $\kappa$-chain and $\lambda$-chain) was additionally performed only in iLCR-negative DLBCL.

\section{Statistical analyses}

The distribution of patient characteristics between iLCRpositive and iLCR-negative DLBCL groups was compared using Fisher's exact test for categorical variables and Wilcoxon's rank sum test for continuous variables. Overall survival (OS) was defined as the time from diagnosis to the last follow-up or death from any cause, and progression-free survival (PFS) was defined as the time from diagnosis to disease progression, relapse, or death from any cause. OS and PFS were estimated by the Kaplan-Meier method and the log-rank test was used to compare the outcomes between groups. A p-value less than 0.05 was considered significant. All statistical analyses were performed with EZR (Saitama Medical Center, Jichi Medical University, Saitama, Japan), which is a statistical software developed with expanded functions of R (The R Foundation for Statistical Computing, Vienna, Austria). ${ }^{17}$ The study protocol was approved by the institutional review board of Osaka City General Hospital in accordance with the Declaration of Helsinki. Informed consent was received in the form of opt-outs on the website of Osaka City General Hospital. Written informed consent was received from the patients whose samples were necessary for the additional immunostaining of $\kappa$ - and $\lambda$-chains.

\section{RESULTS}

We identified 111 patients who underwent FCM and were diagnosed with DLBCL at Osaka City General Hospital. Thirty patients were excluded due to the low proportion ( $\leq$ $50 \%$ ) of CD19 and CD20-positive cells in the gated population in FCM. Thus, 81 patients were finally analyzed. Their baseline characteristics are presented in Table 1. Sixty-three cases of iLCR-positive DLBCL (78\%) and 18 cases of iLCR-negative DLBCL (22\%) were identified (representative cases of iLCR-positive and iLCR-negative DLBCL are shown in Fig. 1), with a median age of 74 years. Of the patients, $46 \%$ were male, $77 \%$ had stage III/IV, and $67 \%$ had a high-intermediate or high IPI. Forty percent of the patients were treated with R-CHOP, $43 \%$ with R-THP$\mathrm{COP}$, and $6 \%$ with rituximab alone. In total, $93 \%$ of the patients received rituximab-containing regimens. Therapeutic regimens did not significantly differ between iLCR-positive and iLCR-negative DLBCL. Although the baseline characteristics did not significantly differ in any clinical category between iLCR-positive and iLCR-negative DLBCL, patients with PS $\geq 2(30.2 \%$ vs $55.6 \%, \mathrm{p}=0.057)$ and stage $\geq$ III $(71.4 \%$ vs $94.4 \%, p=0.057)$ were slightly more frequent in the iLCR-negative DLBCL group than in the iLCR-positive DLBCL group.

The pathological characteristics are summarized in Table 2. A total of 75 samples were available for a retrospective pathological review. Six cases (5 cases of iLCR-positive DLBCL and 1 case of iLCR-negative DLBCL) were unavailable because the samples were transferred to other facilities. Although there were no significant differences in pathological findings between the two groups, necrosis was slightly 
Table 1. Baseline characteristics of the patients with iLCR-positive and iLCR-negative DLBCL

\begin{tabular}{|c|c|c|c|c|}
\hline & $\begin{array}{l}\text { All patients } \\
(\mathrm{n}=81)\end{array}$ & $\begin{array}{l}\text { iLCR-positive DLBCL } \\
\qquad(\mathrm{n}=63)\end{array}$ & $\begin{array}{l}\text { iLCR-negative DLBCL } \\
\quad(\mathrm{n}=18)\end{array}$ & $P$-value \\
\hline Age (year) (median, IQR) & $74(63-79)$ & $73(62-79)$ & $75(69-79)$ & 0.31 \\
\hline Male (\%) & $37(45.7)$ & $30(47.6)$ & $7(38.9)$ & 0.60 \\
\hline PS 2-4 (\%) & $29(35.8)$ & $19(30.2)$ & $10(55.6)$ & 0.057 \\
\hline LDH (U/L) (median, IQR) & $305(223-470)$ & $293(221-440)$ & $345(274-534)$ & 0.14 \\
\hline Stage III-IV (\%) & $62(76.5)$ & $45(71.4)$ & $17(94.4)$ & 0.057 \\
\hline Extranodal lesions $\geq 2(\%)$ & $20(24.7)$ & $15(23.8)$ & $5(27.8)$ & 0.76 \\
\hline Bulky lesion (\%) & $16(19.8)$ & $11(17.5)$ & $5(27.8)$ & 0.33 \\
\hline B-symptoms (\%) & $21(25.9)$ & $14(22.2)$ & $7(38.9)$ & 0.22 \\
\hline IPI (\%) & & & & 0.16 \\
\hline low & $16(19.8)$ & $15(23.8)$ & $1(5.6)$ & \\
\hline low-int & $11(13.6)$ & $9(14.3)$ & $2(11.1)$ & \\
\hline high-int & $22(27.2)$ & $18(28.6)$ & $4(22.2)$ & \\
\hline high & $32(39.5)$ & $21(33.3)$ & $11(61.1)$ & \\
\hline \multicolumn{5}{|l|}{$\operatorname{FCM}(\%)$} \\
\hline CD5+ & $16(19.8)$ & $14(22.2)$ & $2(11.1)$ & 0.50 \\
\hline $\mathrm{CD} 10+$ & $29(35.8)$ & $25(39.7)$ & $4(22.2)$ & 0.27 \\
\hline CD19+ & $77(95.1)$ & $60(95.2)$ & $17(94.4)$ & 1.00 \\
\hline $\mathrm{CD} 20+$ & $75(92.6)$ & $58(92.1)$ & $17(94.4)$ & 1.00 \\
\hline $\mathrm{CD} 23+$ & $10(12.3)$ & $7(11.1)$ & $3(16.7)$ & 0.69 \\
\hline $\mathrm{CD} 25+$ & $29(35.8)$ & $25(39.7)$ & $4(22.2)$ & 0.27 \\
\hline CD30+ & $5(6.2)$ & $2(3.2)$ & $3(16.7)$ & 0.070 \\
\hline Treatment (\%) & & & & 0.30 \\
\hline R-CHOP & $32(39.5)$ & $26(41.3)$ & $6(33.3)$ & \\
\hline R-THP-COP & $35(43.2)$ & 27 (42.9) & $8(44.4)$ & \\
\hline THP-COP & $2(2.5)$ & $2(3.2)$ & $0(0.0)$ & \\
\hline $\mathrm{R}$ & $5(6.2)$ & $3(4.8)$ & $2(11.1)$ & \\
\hline others* & $4(4.9)$ & $4(6.3)$ & $0(0.0)$ & \\
\hline none & $3(3.7)$ & $1(1.6)$ & $2(11.1)$ & \\
\hline
\end{tabular}

Abbreviations: DLBCL, diffuse large B-cell lymphoma; FCM, flow cytometry; iLCR, immunoglobulin light chain restriction; IPI, International Prognostic Index; IQR, interquartile range; LDH, lactate dehydrogenase; PS, performance status.

* Four patients with iLCR-positive DLBCL received the following therapeutic regimens: 2 R-CEOP (rituximab, cyclophosphamide, etoposide, vincristine, and prednisone), 1 R-DeVIC (rituximab, etoposide, dexamethasone, ifosfamide, carboplatin), and 1 prednisone alone.

more frequent in iLCR-negative DLBCL than in iLCR-positive DLBCL (47.1\% vs 20.7\%, p = 0.058) (Table 2) (representative pathological features of iLCR-negative DLBCL are shown in Fig. 2). The degree of mitosis was unable to be evaluated because the number of mitotic cells was difficult to accurately count, resulting in unclear data. Immunohistochemical analysis revealed that BCL6 expression was significantly lower in iLCR-negative DLBCL than in iLCR-positive DLBCL (12.5\% vs 65.5\%, p < 0.001), and CD10 expression was slightly lower in iLCR-negative DLBCL (11.8\% vs $35.0 \%, p=0.078)$. Consequently, non-germinal center B-cell like (non-GCB) type was relatively more frequent in iLCR-negative DLBCL (81.2\% vs $57.6 \%, \mathrm{p}=0.14)$. Immunostaining for $\kappa$ - and $\lambda$-chain proteins was conducted in 13 of 18 iLCR-negative DLBCL cases, but specific expression was not observed.

With a median observation time of 540 days (594 days in iLCR-positive DLBCL and 308 days in iLCR-negative
DLBCL), the 3-year PFS of iLCR-positive and iLCR-negative DLBCL was $49.4 \%$ and $56.6 \%(p=0.75)($ Fig. $3 \mathrm{~A})$, and the 3 -year OS was $60.5 \%$ and $60.2 \%(p=0.64)$ (Fig. 3B), respectively. There were no significant differences in the PFS or OS between iLCR-positive and iLCR-negative DLBCL according to the presence of necrosis and cellular origin (Fig. 3C-F).

\section{DISCUSSION}

This retrospective study investigated the clinicopathological characteristics of recently diagnosed iLCR-negative DLBCL compared with iLCR-positive DLBCL, as rituximab was introduced as the standard frontline chemotherapy for patients with DLBCL. Although patients with iLCR-negative DLBCL had a slightly poorer clinical background in terms of poor PS and advanced stage, survival outcomes were equivalent between the two groups. Pathological analysis 

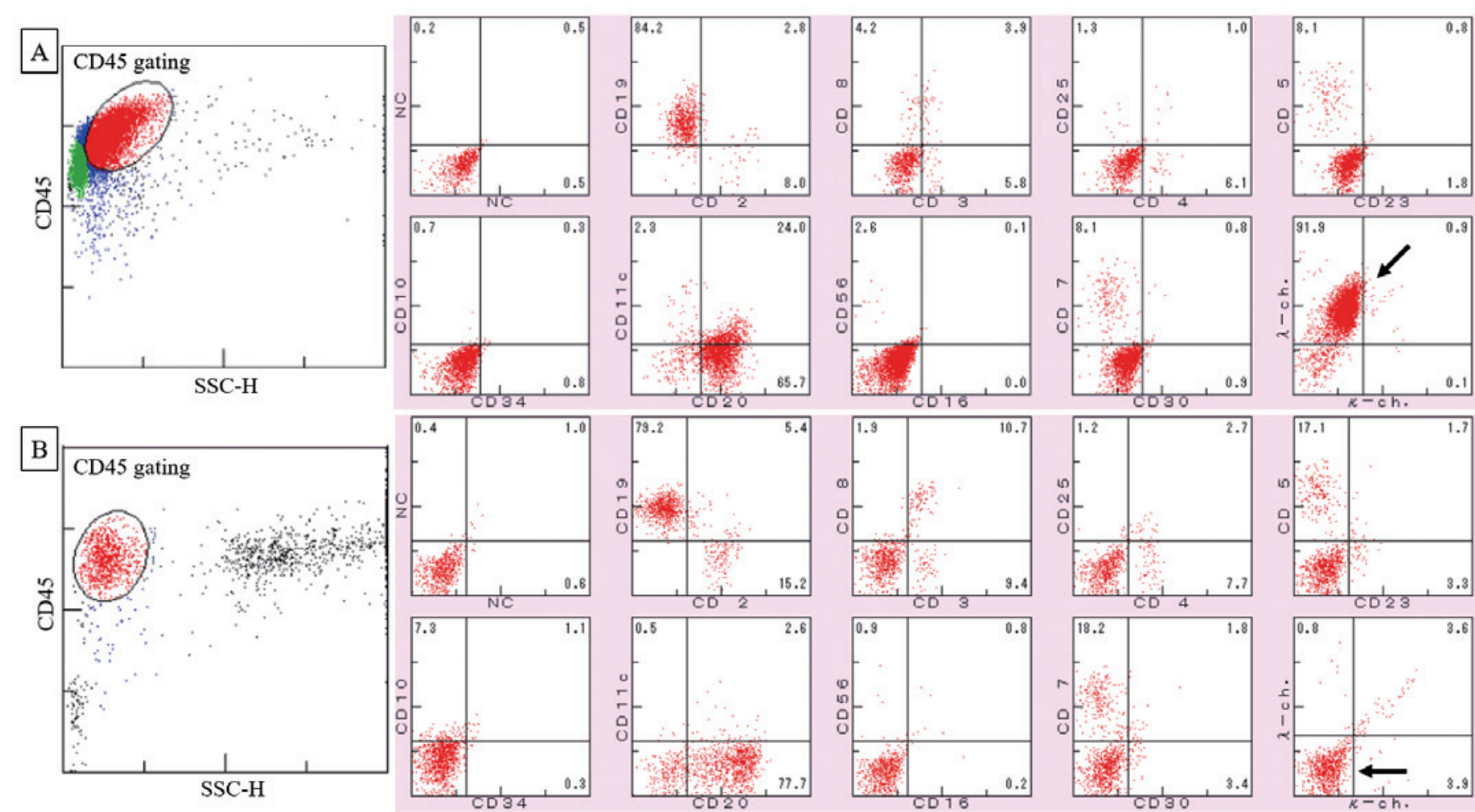

Fig. 1. The flow cytometric analysis of two representative cases of immunoglobulin light chain restriction (iLCR)-positive and iLCR-negative diffuse large B-cell lymphoma (DLBCL). The black arrows indicate the population of lymphoma cells. Both types of lymphoma cells significantly expressed B-cell markers of CD19 and CD20. iLCR-positive DLBCL $(\boldsymbol{A})$ showed $\lambda$ chain dominant light chain restriction $(\kappa 1.0 \%$ $<<\lambda$ 92.8\%), although iLCR-negative DLBCL $(\boldsymbol{B})$ lacked such restriction $(\kappa 7.5 \%, \lambda 4.4 \%)$.

revealed that necrosis was more frequent in iLCR-negative DLBCL, and the expression of germinal center B-cell markers (BCL6 and CD10) was lower in iLCR-negative DLBCL, resulting in a higher frequency of non-GCB type in iLCRnegative DLBCL than in iLCR-positive DLBCL.

Only one retrospective study examined the clinical features of iLCR-negative DLBCL in comparison with iLCRpositive DLBCL, and demonstrated that the patient characteristics and prognosis were similar between the two groups. ${ }^{3}$ Our results differed in that iLCR-negative DLBCL patients had a slightly poorer clinical background, but were consistent in that iLCR-negativity did not affect the clinical outcomes. The study also revealed that CD10 on FCM was less frequent in iLCR-negative DLBCL, ${ }^{3}$ which was consistent with our immunohistochemistry data. Another retrospective study investigated 36 cases of iLCR-negative DLBCL without the control of iLCR-positive DLBCL. ${ }^{5}$ In this study, CD10, BCL6, and MUM1 were positive in 3 of 24 (12.5\%), 11 of 17 (64.7\%), and 12 of 17 (70.6\%) iLCR-negative DLBCL cases, respectively, which translated into a non-GCB subtype in 13 of $18(72.2 \%)$ patients with available data. ${ }^{5}$ The relatively lower expression of CD10 and higher frequency of non-GCB subtype in iLCR-negative DLBCL are consistent with our study, but lower expression of BCL6 was not observed. ${ }^{5}$ The non-GCB subtype has been reported to have a poorer prognosis than the GCB subtype ${ }^{16}$ therefore, the dominance of non-GCB subtype in iLCR-negative DLBCL may partly explain the poor baseline conditions in patients with
iLCR-negative DLBCL. Moreover, a recent study reported that rituximab-containing chemotherapy improved the survival of the non-GCB group and counteracted its negative impact on survival in DLBCL,${ }^{18}$ which can also explain the absence of difference in clinical outcomes between iLCRpositive and iLCR-negative DLBCL in our study.

Pathological findings associated with aggressive tumor cell proliferation, including necrosis, apoptosis, and mitosis, may be factors affecting the decrease in the detectability of iLCR on FCM in B-cell lymphoma. ${ }^{8,19}$ Our study confirmed the high frequency of histological necrosis in iLCR-negative DLBCL, but the degree of apoptosis and other pathological findings did not correlate with iLCR-negativity. This suggested that necrosis is a pathological feature strongly associated with iLCR-negativity. The detectability of iLCR by FCM depends on the pathological subtype of B-cell lymphoma. In previous reports, iLCR-negativity was observed in $22 \%-30 \%$ of DLBCL, $6 \%-7 \%$ of follicular lymphoma, $4 \%-30 \%$ of marginal zone lymphoma, $0 \%-4 \%$ of mantle cell lymphoma, $0 \%-6 \%$ of chronic lymphocytic lymphoma, and $7 \%-19 \%$ of overall B-cell NHL. ${ }^{3-6}$ The ratio of iLCR-negative DLBCL in the present study (22\%) was consistent with these published results. The detectability of iLCR was slightly lower in aggressive B-cell lymphoma than in lowgrade B-cell lymphoma, which partially reflects the number of cells available for FCM analysis. ${ }^{20}$ The cellular viability is inversely proportional to the degree of necrosis in lymphoma cells. Vallangeon et al. examined 93 DLBCL 
Table 2. Pathological characteristics of the patients with iLCR-positive and iLCR-negative DLBCL

\begin{tabular}{|c|c|c|c|c|}
\hline & $\begin{array}{l}\text { All patients } \\
\quad(\mathrm{n}=81)\end{array}$ & $\begin{array}{l}\text { iLCR-positive DLBCL } \\
\qquad(\mathrm{n}=63)\end{array}$ & $\begin{array}{l}\text { iLCR-negative DLBCL } \\
\qquad(\mathrm{n}=18)\end{array}$ & $P$-value \\
\hline Pathological type (\%)* & & & & 0.58 \\
\hline centroblastic & $70(93.3)$ & $53(91.4)$ & $17(100.0)$ & \\
\hline immunoblastic & $5(6.7)$ & $5(8.6)$ & $0(0.0)$ & \\
\hline Nucleolus (\%)* & & & & 0.47 \\
\hline multiple & $53(70.7)$ & $39(67.2)$ & $14(82.4)$ & \\
\hline single centrally located & $5(6.7)$ & $5(8.6)$ & $0(0.0)$ & \\
\hline small unclear & $17(22.7)$ & $14(24.1)$ & $3(17.6)$ & \\
\hline Cellularity (\%)* & & & & 0.50 \\
\hline moderate & $59(78.7)$ & $47(81.0)$ & $12(70.6)$ & \\
\hline high & $16(21.3)$ & $11(19.0)$ & $5(29.4)$ & \\
\hline Apoptosis (\%)* & & & & 0.31 \\
\hline absent & $6(8.0)$ & $4(6.9)$ & $2(11.8)$ & \\
\hline occasional & $38(50.7)$ & $32(55.2)$ & $6(35.3)$ & \\
\hline abundant & $31(41.3)$ & $22(37.9)$ & $9(52.9)$ & \\
\hline Necrosis (\%)* & & & & 0.058 \\
\hline absent & $55(73.3)$ & $46(79.3)$ & $9(52.9)$ & \\
\hline present & $20(26.7)$ & $12(20.7)$ & $8(47.1)$ & \\
\hline \multicolumn{5}{|l|}{ Immunohistochemistry $\dagger^{\dagger}$} \\
\hline $\mathrm{CD} 10+$ & 23/77 (29.9) & $21 / 60(35.0)$ & 2/17 (11.8) & 0.078 \\
\hline $\mathrm{CD} 20+$ & $81 / 81(100.0)$ & $63 / 63(100.0)$ & $18 / 18(100.0)$ & $\mathrm{NE}$ \\
\hline BCL2+ & $31 / 77(40.3)$ & $27 / 60(45.0)$ & $4 / 17(23.5)$ & 0.16 \\
\hline BCL6+ & $40 / 74(54.1)$ & $38 / 58(65.5)$ & $2 / 16(12.5)$ & $<0.001$ \\
\hline MUM1+ & $41 / 74(55.4)$ & $31 / 58(53.4)$ & $10 / 16(62.5)$ & 0.58 \\
\hline MYC+ & $11 / 75(14.7)$ & $9 / 58(15.5)$ & 2/17 (11.8) & 1.00 \\
\hline Cell of Origin $\ddagger$ & & & & 0.14 \\
\hline $\mathrm{GCB}$ & $28(37.3)$ & $25(42.4)$ & $3(18.8)$ & \\
\hline non-GCB & $47(62.7)$ & $34(57.6)$ & $13(81.2)$ & \\
\hline
\end{tabular}

* Six cases ( 5 cases of iLCR-positive DLBCL and 1 case of iLCR-negative DLBCL) were not available for retrospective pathological review because the samples were transferred to other facilities.

$\uparrow$ Denominators indicate total number of cases available for each immunohistochemical analysis.

$\$$ Six cases (4 cases of iLCR-positive DLBCL and 2 cases of iLCR-negative DLBCL) were missing.

Abbreviations: DLBCL, diffuse large B-cell lymphoma; GCB, germinal center B-cell-like; non-GCB, non-germinal center B-cell-like; iLCR, immunoglobulin light chain restriction; NE, not evaluable.

samples using several FCM techniques and revealed that necrosis was more frequent in iLCR-negative DLBCL than in iLCR-positive DLBCL on pathological examination; 13 of 27 (48.1\%) patients with iLCR-negative DLBCL and 10 of 66 $(15.2 \%)$ patients with iLCR-positive DLBCL had necrosis. ${ }^{21}$ The frequency of histological necrosis in iLCR-positive and iLCR-negative DLBCL was consistent with our study. We assumed that the higher frequency of histological necrosis derived from the biologically aggressive non-GCB subtype of DLBCL reduced the detectability of iLCR on FCM, but there was no correlation between the presence of necrosis and cellular origin; necrosis was present in 7 of 27 (25.9\%) cases of the GCB subtype and 13 of 47 cases of the non-GCB subtype (27.7\%) (Fisher's exact test demonstrated no significance, $\mathrm{p}=1.00)$. Although the underlying mechanism is unclear, tumor necrosis and non-GCB subtype may play different roles in the loss of iLCR.

Tumor necrosis defined as the hypo-metabolism area on positron emission tomography-computed tomography
(PET-CT) was reported to be associated with a poorer prognosis in patients with DLBCL treated with R-CHOP chemotherapy. ${ }^{22}$ Thus, an inferior prognosis of iLCR-negative DLBCL was preconceived because more than $40 \%$ of patients with iLCR-negative DLBCL had histological necrosis. However, histological necrosis did not have an adverse effect on clinical outcomes in both iLCR-positive and iLCRnegative DLBCL (Fig. 3C, D). A possible explanation for this disassociation is the poor concordance between histologically confirmed necrosis and radiologically diagnosed necrosis on PET-CT. ${ }^{23}$ Different from radiological necrosis, histological necrosis may not be a poor prognostic factor for DLBCL.

The reason for the lack of iLCR in DLBCL other than necrosis remains unclear. Previous studies proposed abnormalities of gene transcription or the translocation of fully assembled proteins on the cell surface. ${ }^{7,24} \mathrm{Li}$ et al. reported that iLCR-negative B-cell NHLs harbored clonal immunoglobulin heavy chain rearrangements as frequently as 


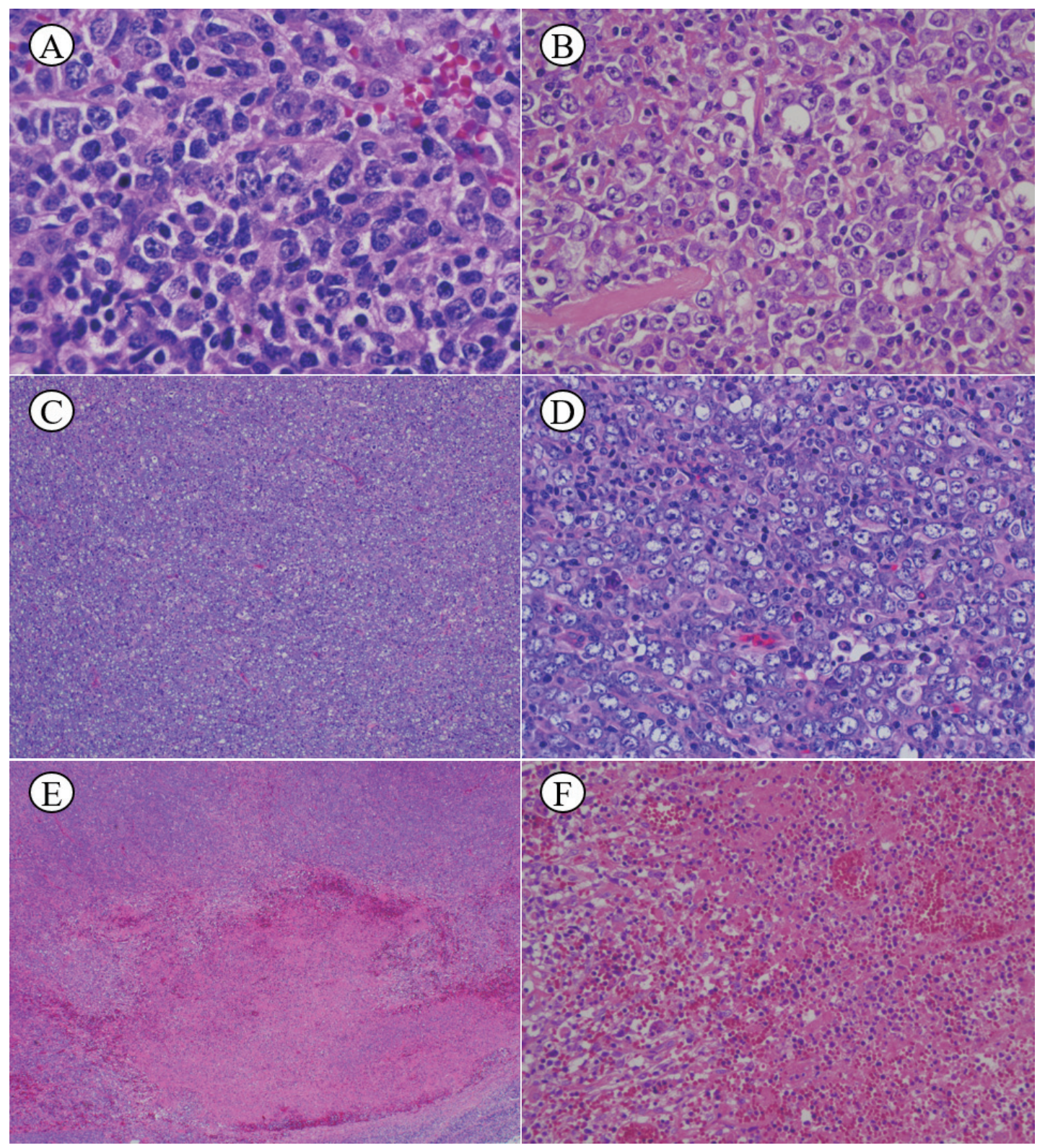

Fig. 2. Pathological findings observed in diffuse large B-cell lymphoma (DLBCL) without immunoglobulin light chain restriction (iLCR); a case exhibiting a centroblastic variant with vesicular chromatin and prominent multiple nucleoli $(\boldsymbol{A})(\times 600)$; a case with abundant apoptotic cells $(\boldsymbol{B})(\times 400)$; a case demonstrating high cellularity $(\boldsymbol{C}: \times 100)(\boldsymbol{D}: \times 400)$; and a case with geographic necrosis $(\boldsymbol{E}: \times 40)(\boldsymbol{F}: \times 200)$.

iLCR-positive B-cell NHL, which suggested the existence of monoclonality and defective protein synthesis or intracellular protein transport as a mechanism for the lack of iLCR. ${ }^{9}$ Although the lack of expression of surface light chains on a mature B-cell population suggests a neoplastic process, it should not be the only parameter used for diagnosing B-cell lymphoma, as this is also observed in non-neoplastic conditions, such as follicular hyperplasia, albeit rarely. ${ }^{4,9,24}$

FCM analysis for cytoplasmic immunoglobulin light chains may increase the sensitivity of iLCR in B-cell NHL; ${ }^{25}$ therefore, we further evaluated the cytoplasmic immunoglobulin light chain expression in iLCR-negative DLBCL by immunohistochemical staining. However, it was unavailable. As previously reported, the significant expression of light chain proteins or light chain restriction was not detected, probably because of the small amount of light chain proteins on lymphoma cells without plasmacytic differentiation and high extracellular background. ${ }^{26}$ In situ hybridization for intra-cytoplasmic immunoglobulin light chain mRNA may be an alternative tool to detect light chain restriction more accurately because of the absence of interstitial

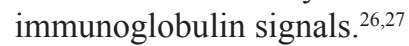

Several limitations of the present study should be acknowledged. First, although all consecutive patients were evaluated during the study period, the retrospective design at a single institute was subject to selection bias. Second, the small number of patients recruited may have reduced the statistical power of the analysis. Third, we were unable to 
A

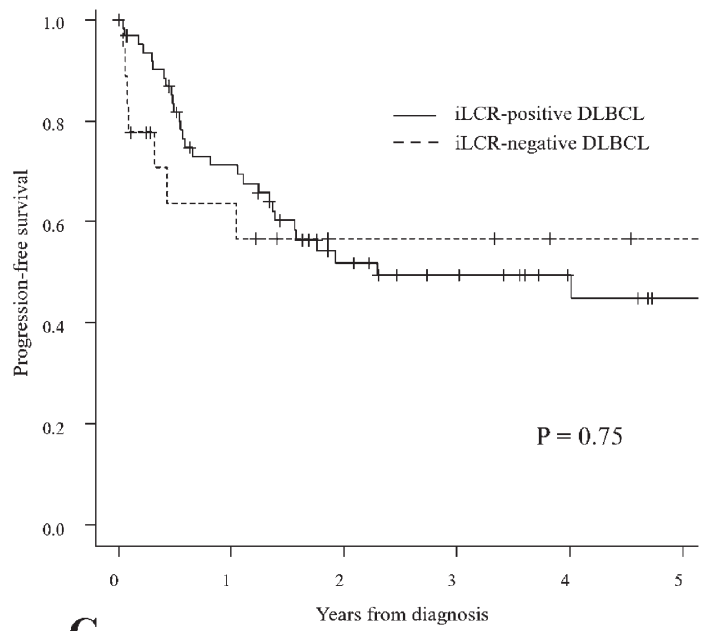

C

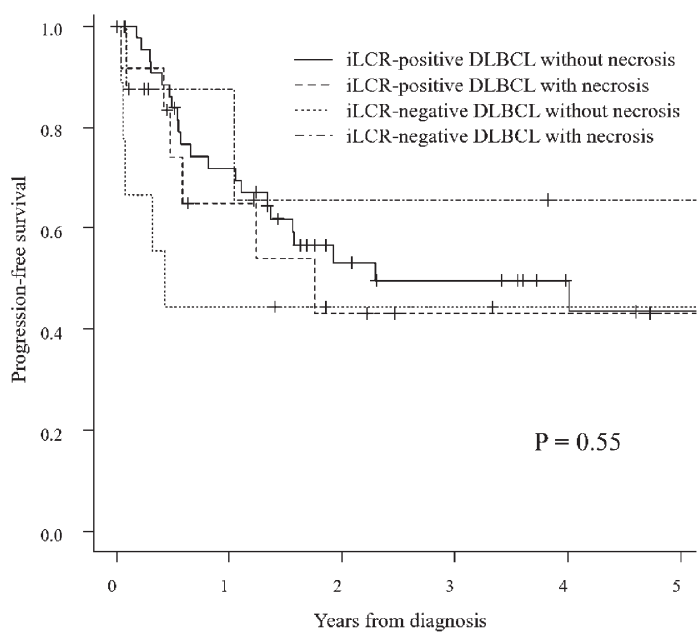

$\mathbf{E}$

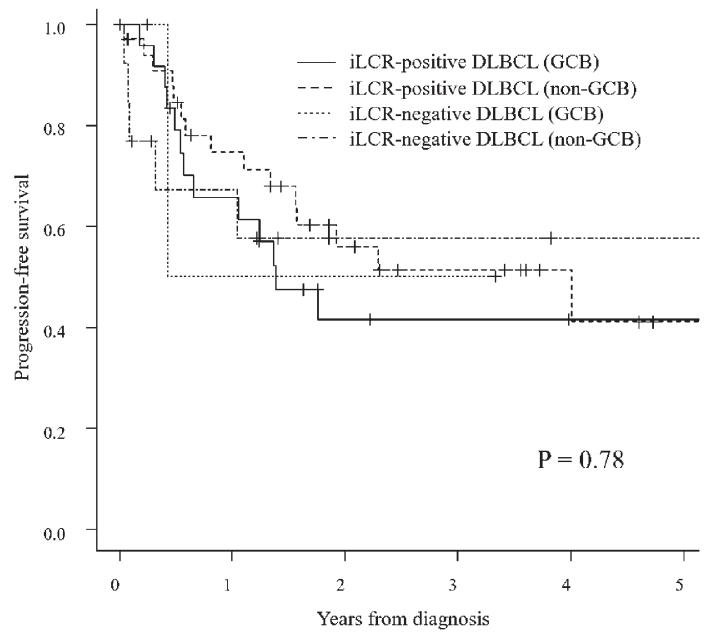

B

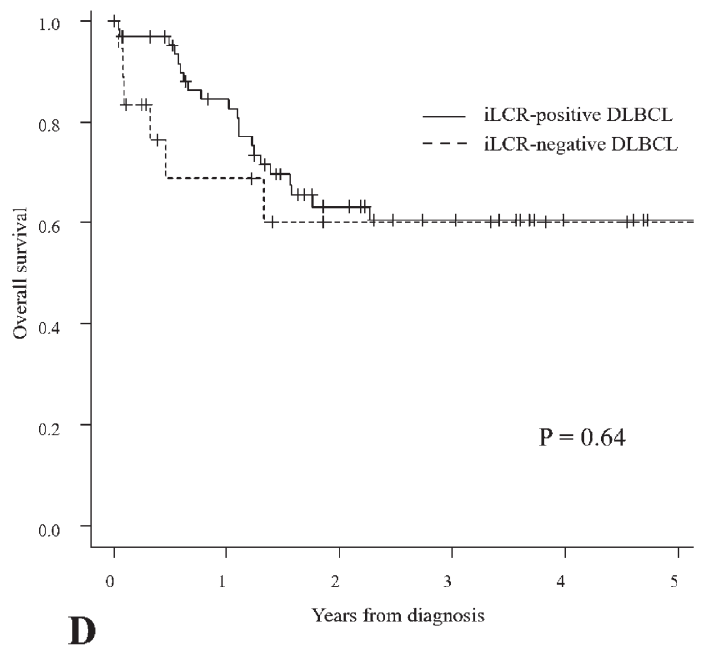

D

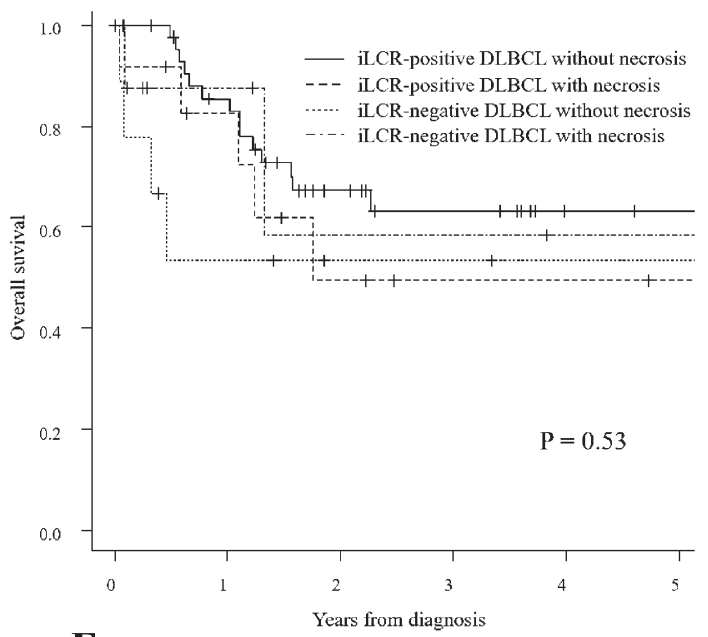

$\mathbf{F}$

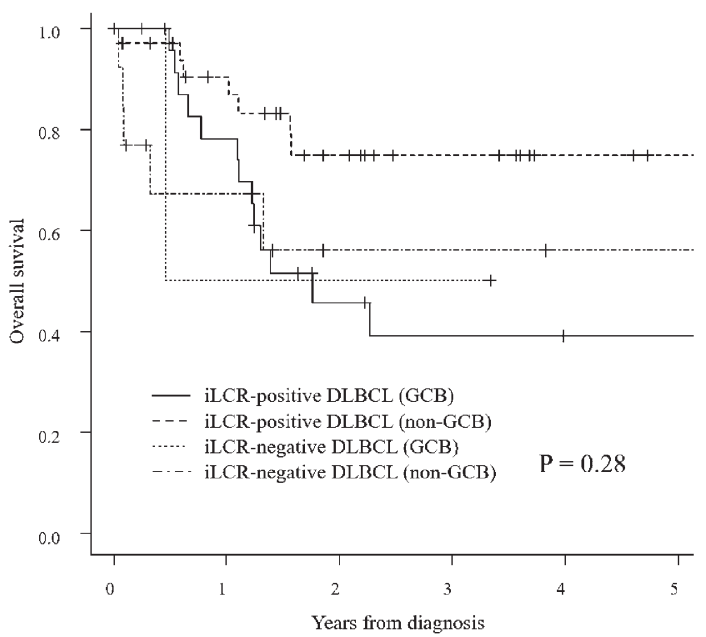

Fig. 3. Progression-free survival (PFS) and overall survival (OS) of immunoglobulin light chain restriction (iLCR)-positive and negative DLBCL, and those according to the presence of necrosis and cell of origin. The 3 -year PFS of iLCRpositive and iLCR-negative DLBCL was $49.4 \%$ and $56.6 \%(\mathrm{p}=0.75)(A)$, and the 3 -year OS was $60.5 \%$ and $60.2 \%(\mathrm{p}$ $=0.64$ ), respectively $(\boldsymbol{B})$. The 3-year PFS of iLCR-positive DLBCL with or without necrosis was $43.2 \%$ and $49.7 \%$, and that of iLCR-negative DLBCL with or without necrosis was $65.6 \%$ and $44.4 \%(C)(p=0.55)$, respectively. The 3-year OS of iLCR-positive DLBCL with or without necrosis was $49.5 \%$ and $63.2 \%$, and that of iLCR-negative DLBCL with or without necrosis was $58.3 \%$ and $53.3 \%(\boldsymbol{D})(\mathrm{p}=0.53)$, respectively. The 3 -year PFS of iLCR-positive DLBCL of the GCB or non-GCB subtype was $41.6 \%$ and $51.3 \%$, and that of iLCR-negative DLBCL of the GCB or non-GCB subtype was $50.0 \%$ and $57.7 \%(\boldsymbol{E})(\mathrm{p}=0.78)$, respectively. The 3-year OS of iLCR-positive DLBCL of the GCB or non-GCB subtype was $39.2 \%$ and $74.8 \%$, and that of iLCR-negative DLBCL of the GCB or non-GCB subtype was $50.0 \%$ and $56.1 \%(F)(\mathrm{p}=0.28)$, respectively. There were no significant differences among these groups. 
investigate the rearrangement status of MYC, BCL2, and BCL6 by fluorescence in situ hybridization, which may have revealed some DLBCL cases to be high-grade B-cell lymphoma with MYC and BCL2 and/or BCL6 rearrangements (HGBL-DH/TH) according to the current WHO classification. However, HGBL-DH/TH accounts for less than $8 \%$ of DLBCL cases and only one patient in this study presented with high-risk immunological features of HGBL-DH/TH: GCB subtype with dual protein expression of MYC and BCL2. ${ }^{28,29}$

In conclusion, our study revealed that the clinical prognosis of patients with iLCR-negative DLBCL is similar to that of patients with iLCR-positive DLBCL. Tumor necrosis and lower expression of germinal center markers may be specific pathological features of iLCR-negative DLBCL; however, the biological basis for these characteristics and the loss of iLCR in DLBCL was unclear. Further studies are warranted to clarify the underlying relationships.

\section{ACKNOWLEDGMENTS}

We would like to express our gratitude to the laboratory technicians of the pathology section at Osaka City General Hospital for the additional preparation of pathological specimens.

\section{AUTHOR CONTRIBUTIONS}

YN and MY analyzed the clinical data and drafted the manuscript, Figures, and Tables. MS and TI evaluated the pathological characteristics. KS, NY, MT, TY, YH, TN, MH, and TY cooperated in treatment and evaluated the clinical data.

\section{CONFLICT OF INTEREST}

The authors declare that they have no conflicts of interest.

\section{REFERENCES}

1 Seegmiller AC, Hsi ED, Craig FE. The current role of clinical flow cytometry in the evaluation of mature B-cell neoplasms. Cytometry B Clin Cytom. 2019; 96: 20-29.

2 Menon MP, Pittaluga S, Jaffe ES. The histological and biological spectrum of diffuse large B-cell lymphoma in the World Health Organization classification. Cancer J. 2012; 18: 411-420.

3 Tomita N, Takeuchi K, Hyo R, et al. Diffuse large B cell lymphoma without immunoglobulin light chain restriction by flow cytometry. Acta Haematol. 2009; 121: 196-201.

4 Horna P, Olteanu H, Kroft SH, Harrington AM. Flow cytometric analysis of surface light chain expression patterns in B-cell lymphomas using monoclonal and polyclonal antibodies. Am J Clin Pathol. 2011; 136: 954-959.

5 Matsushita H, Nakamura N, Tanaka Y, et al. Clinical and pathological features of B-cell non-Hodgkin lymphomas lacking the surface expression of immunoglobulin light chains. Clin Chem Lab Med. 2012; 50: 1665-1670.
6 Ohmoto A, Maeshima AM, Taniguchi H, et al. Histopathological analysis of B-cell non-Hodgkin lymphomas without light chain restriction by using flow cytometry. Leuk Lymphoma. 2015; 56: 3301-3305.

7 Kaleem Z, Zehnbauer BA, White G, Zutter MM. Lack of expression of surface immunoglobulin light chains in B-cell non-Hodgkin lymphomas. Am J Clin Pathol. 2000; 113: 399-405.

8 Bertram HC, Check IJ, Milano MA. Immunophenotyping large B-cell lymphomas. Flow cytometric pitfalls and pathologic correlation. Am J Clin Pathol. 2001; 116: 191-203.

9 Li S, Eshleman JR, Borowitz MJ. Lack of surface immunoglobulin light chain expression by flow cytometric immunophenotyping can help diagnose peripheral B-cell lymphoma. Am J Clin Pathol. 2002; 118: 229-234.

10 International Non-Hodgkin's Lymphoma Prognostic Factors Project. A predictive model for aggressive non-Hodgkin's lymphoma. N Engl J Med. 1993; 329: 987-994.

11 Yoshida M, Nakao T, Horiuchi M, et al. Analysis of elderly patients with diffuse large B-cell lymphoma: aggressive therapy is a reasonable approach for 'unfit' patients classified by comprehensive geriatric assessment. Eur J Haematol. 2016; 96: 409-416.

12 Hara T, Yoshikawa T, Goto H, et al. R-THP-COP versus $\mathrm{R}-\mathrm{CHOP}$ in patients younger than 70 years with untreated diffuse large B cell lymphoma: A randomized, open-label, noninferiority phase 3 trial. Hematol Oncol. 2018; 36: 638-644.

13 Borowitz MJ, Guenther KL, Shults KE, Stelzer GT. Immunophenotyping of acute leukemia by flow cytometric analysis. Use of CD45 and right-angle light scatter to gate on leukemic blasts in three-color analysis. Am J Clin Pathol. 1993; 100: 534-540.

14 Geary WA, Frierson HF, Innes DJ, Normansell DE. Quantitative criteria for clonality in the diagnosis of B-cell non-Hodgkin's lymphoma by flow cytometry. Mod Pathol. 1993; 6: 155-161.

15 Swerdlow SH, Campo E, Pileri SA, et al. The 2016 revision of the World Health Organization classification of lymphoid neoplasms. Blood. 2016; 127: 2375-2390.

16 Hans CP, Weisenburger DD, Greiner TC, et al. Confirmation of the molecular classification of diffuse large B-cell lymphoma by immunohistochemistry using a tissue microarray. Blood. 2004; 103: 275-282.

17 Kanda Y. Investigation of the freely available easy-to-use software 'EZR' for medical statistics. Bone Marrow Transplant. 2013; 48: 452-458.

18 Seki R, Ohshima K, Fujisaki T, et al. Prognostic impact of immunohistochemical biomarkers in diffuse large B-cell lymphoma in the rituximab era. Cancer Sci. 2009; 100: 1842-1847.

19 Jennings CD, Foon KA. Recent advances in flow cytometry: application to the diagnosis of hematologic malignancy. Blood. 1997; 90: 2863-2892.

20 Zander DS, Iturraspe JA, Everett ET, Massey JK, Braylan RC. Flow cytometry. In vitro assessment of its potential application for diagnosis and classification of lymphoid processes in cytologic preparations from fine-needle aspirates. Am J Clin Pathol. 1994; 101: 577-586.

21 Vallangeon BD, Tyer C, Williams B, Lagoo AS. Improved 
detection of diffuse large B-cell lymphoma by flow cytometric immunophenotyping-Effect of tissue disaggregation method. Cytometry B Clin Cytom. 2016; 90: 455-461.

22 Song MK, Chung JS, Shin DY, et al. Tumor necrosis could reflect advanced disease status in patients with diffuse large $\mathrm{B}$ cell lymphoma treated with R-CHOP therapy. Ann Hematol. 2017; 96: 17-23.

23 Kahle XU, Hovingh M, Noordzij W, et al. Tumour necrosis as assessed with ${ }^{18} \mathrm{~F}$-FDG PET is a potential prognostic marker in diffuse large B cell lymphoma independent of MYC rearrangements. Eur Radiol. 2019; 29: 6018-6028.

24 Zhao XF, Cherian S, Sargent R, et al. Expanded populations of surface membrane immunoglobulin light chain-negative B cells in lymph nodes are not always indicative of B-cell lymphoma. Am J Clin Pathol. 2005; 124: 143-150.

25 Bardales RH, Al-Katib AM, Carrato A, Koziner B. Detection of intracytoplasmic immunoglobulin by flow cytometry in B-cell malignancies. J Histochem Cytochem. 1989; 37: 83-89.
26 Rimsza LM, Day WA, McGinn S, et al. Kappa and lambda light chain mRNA in situ hybridization compared to flow cytometry and immunohistochemistry in B cell lymphomas. Diagn Pathol. 2014; 9: 144.

27 Beck RC, Tubbs RR, Hussein M, Pettay J, Hsi ED. Automated colorimetric in situ hybridization (CISH) detection of immunoglobulin (Ig) light chain mRNA expression in plasma cell (PC) dyscrasias and non-Hodgkin lymphoma. Diagn Mol Pathol. 2003; 12: 14-20.

28 Copie-Bergman C. Double-hit DLBCL: should we limit FISH testing? Blood. 2018; 131: 1997-1998.

29 Scott DW, King RL, Staiger AM, et al. High-grade B-cell lymphoma with MYC and BCL2 and/or BCL6 rearrangements with diffuse large B-cell lymphoma morphology. Blood. 2018; 131: 2060-2064. 